\title{
Evidence of Retinal Degeneration in Wolfram Syndrome
}

Matteo Scaramuzzi ${ }^{1,2}$ MD, Priyanka Kumar ${ }^{3}, \mathrm{MD}$, Neal Peachey $\mathrm{PhD}^{2}$, Paolo Nucci MD, FEBO $^{1}$, Elias I. Traboulsi MD, $\mathrm{MEd}^{2}$

${ }^{1}$ Department of Clinical Sciences and Community Health, Istituto di Ricovero e Cura a Carattere Scientifico (IRCCS) Multimedica, University of Milan, Eye Clinic San Giuseppe Hospital, Via San Vittore, 12, 20123, Milan, Italy

${ }^{2}$ Cole Eye Institute, Cleveland Clinic, 9500 Euclid Avenue, 41195, Cleveland, OH, USA.

${ }^{3}$ Department of Ophthalmology, the Children's Hospital of Philadelphia, Philadelphia, PA, USA. E-mail:

matteo_scaramuzzi@hotmail.com

kumarp1@email.chop.edu

neal.peachey@va.gov

paolo.nucci@,unimi.it

traboue@,ccf.org 
Corresponding author:

Dr. Elias I. Traboulsi

9500 Euclid Avenue, i-20

Cleveland, OH 41195

\author{
traboue@ccf.org
}

$216-445-4363$

This work not been published elsewhere, nor has it been submitted simultaneously for publication elsewhere. 


\section{$\underline{\text { Abstract }}$}

Purpose: Wolfram syndrome is characterized by early onset diabetes mellitus, diabetes insipidus, deafness and optic atrophy, but retinal degeneration has not been described as a major component of the phenotype. We present two cases with Wolfram syndrome and evidence of retinal degeneration.

Materials and Methods: Observational case series. Patients underwent complete ocular examinations as well as retinal imaging and electroretinography.

Results: Both patients had electroretinographic evidence of retinal dysfunction/degeneration in addition to optic atrophy with an otherwise normal-appearing retina.

Conclusions: Some patients with Wolfram syndrome have a mild retinal degeneration that may be a manifestation of the neuronal involvement that is present in this condition.

Keywords: Wolfram syndrome, optic atrophy, retinal degeneration, electroretinography, diabetes mellitus, diabetes insipidus 


\section{Introduction}

Wolfram syndrome is a rare autosomal recessive disorder characterized by diabetes insipidus, sensorineural hearing loss, and neurodegenerative features such as cerebellar ataxia and brainstem atrophy ${ }^{1-5}$. However, only $75 \%$ of patients with Wolfram show the full clinical phenotype ${ }^{1-5}$. The main diagnostic criterion is the combination of optic atrophy and the onset of diabetes mellitus before the age of 16 years ${ }^{1,6}$. In a natural progression study of this disease, the authors argue that the positive diagnostic predictive value of the combination of bilateral optic atrophy and diabetes is $83 \%{ }^{6}$. The syndrome has been linked to mutations in the Wolfram syndrome gene (WFS1), which encodes an endoplasmic reticulum membrane protein, Wolframin ${ }^{7}$. Greater than $90 \%$ of patients with Wolfram syndrome have mutations in $W F S 1^{8}$. We present two patients with Wolfram syndrome with mutations in WFS1 who demonstrate evidence of retinal degeneration/dysfunction, in addition to optic atrophy, diabetes mellitus, and diabetes insipidus.

\section{Materials and methods}

Observational case series of two patients identified upon chart review of all cases of Wolfram syndrome examined between 2000 and 2017 with positive genetic testing and abnormal electroretinographic findings. During the study period, we observed 7 cases of Wolfram syndrome. Among these, we have included the patients with positive genetic testing and abnormal electroretinographic findings. We have performed ERG on another 9 year-old boy, and found it to be normal. No ERG was performed in the other 4 patients. The study was approved by the Cleveland Clinic IRB and patients consented to participate. A comprehensive ophthalmic examination was performed including best-corrected visual acuity, cycloplegic refraction, ocular motility, stereopsis, color vision testing using Ishihara plates (38 Plates Ishihara Booklet Edition 2017, Kanehara Trading In., Tokyo, Japan), direct and consensual pupillary light reflexes, slit-lamp 
biomicroscopy, fundus examination, Goldmann visual field testing, optical coherence tomography (OCT)

of the optic nerves and retina (Cirrus HD-OCT Version 8.0 software,, Carl Zeiss Meditec, Jena, Germany), electroretinography (ERG) (LKC Technologies, Gaithersburg, MD) and molecular genetic testing (CLIA certified laboratory).

\section{$\underline{\text { Results }}$}

\section{Case One:}

A 22 year-old woman was examined before beginning a laboratory technician job that required good color discrimination. She had been diagnosed with type I diabetes at the age of 7 , and had subsequently also developed an overactive bladder, as consequence of bladder neuropathy, treated with solifenacin. Best corrected visual acuity was 20/25 in the right eye and 20/40 in the left eye. Her refraction was $+1.00 \times 20$ in OD and $+0.50+1.00 \times 140$ in OS. She could identify only the test plate in OD and only $8 / 11$ in OS. There was no afferent pupillary defect in either eye. The anterior segment of both eyes was normal. There was mild disc pallor in both eyes, but the fundus appeared otherwise normal, except for a low grade of vessels' narrowing (Figure 1). An MRI of the brain and the orbits with contrast had been obtained and was normal. Light-adapted 0 ERG and light-adapted $30 \mathrm{~Hz}$ Flicker were reduced in amplitude. The dark-adapted 24 ERG and darkadapted 0 ERG were also decreased in amplitude (Figure 2). Goldmann visual fields demonstrated marked bilateral circumferential constriction. The Macula OCT looks normal and the Optic Nerve OCT shows diffuse Nerve Fiber Layer loss in both eyes. Genetic testing was obtained for autosomal dominant optic atrophy, and Wolfram syndrome, although the suspicion for the latter was high in the setting of diabetes mellitus and diabetes insipidus. Two mutations in WFS1 were identified. One was a known pathogenic mutation, c.2648_2651delTCTT; p. 
F883fs*, and the second, a novel likely pathogenic mutation, c.757 A>T; p.K253X, that produces a truncated protein at amino acid 253.

Case Two:

A 26 year-old man was referred in with a known diagnosis of Wolfram syndrome. He carried known pathogenic homozygous mutations in exon 8 of the WFS1 gene,

c.1441_1447dupCTGA; p.Val483Ala. The parents were first cousins. The patient had presented to an ophthalmologist with decreased vision after head trauma at age 6 and the vision loss was attributed to the injury. One year after vision decreased further and the patient developed diabetes mellitus and diabetes insipidus. Best corrected visual acuity was 20/160 in the right eye and 20/320 in the left eye and has remained stable. The patient had moderately high myopia: OD $-8.25+4.25 \times 95$, OS $-9.25+5.75 \times 85$. Anterior segment exam was unremarkable initially, but he gradually developed mild cataracts. Similar to case 1, ophthalmoscopy was notable only for marked disc pallor, without fundus abnormalities (Figure 3). The ERG demonstrated significantly attenuated cone responses with absence of b-wave on light-adapted 0 ERG. Rod responses were also markedly decreased in amplitude on dark-adapted -24 ERG and darkadapted 0 ERG (Figure 2). Goldmann visual fields demonstrated marked bilateral circumferential constriction.

\section{$\underline{\text { Discussion }}$}

Wolfram syndrome is a complex autosomal recessive disorder in which patients present with optic atrophy in the setting of non-autoimmune juvenile diabetes, and progress to develop 
deafness, renal failure and neurologic dysfunction ${ }^{1,6}$. A large proportion of patients suffer from psychiatric and neurologic problems, and in most patients death occurs early, often because of respiratory failure secondary to brain atrophy ${ }^{8}$. It is for all these reasons as well as for genetic counseling that early diagnosis is critical. Our goal is to broaden the understanding of the clinical spectrum of this condition and include retinal degeneration to the list of ophthalmic findings. There have been a few reported cases with abnormal ERGs, but this has not been well explained ${ }^{1}$.

Gunn et al described four patients in 1976 with juvenile diabetes mellitus, optic atrophy, sensorineural hearing loss, and diabetes insipidus, and one of these patients demonstrated granular changes in the macula with a normal ERG, while the rest had normal fundi with ERG abnormalities ${ }^{9}$. Of these three patients, the first was a 17 year-old girl with elevated ERG threshold and diminished amplitude in dark-adapted ERG, a normal cone response, diminished dark-adapted oscillatory potential and abnormal dark adaptation; the second was also a 17 yearold girl with an elevated ERG threshold and diminished amplitudes of dark- and light-adapted responses, diminished dark-adapted oscillatory potential and abnormal dark adaptation; the third patient was a 17 year-old boy with elevated threshold and reduced cone response amplitudes 9 Rose et al published a series of seven patients in 1966 with juvenile diabetes and optic atrophy, of whom two demonstrated fine granular changes to the retina. Electroretinography was normal in one patient, and abnormal in the second ${ }^{10}$. Of note, in this series, the diagnosis was unclear, as patients had findings compatible with Friedreich ataxia as well as Refsum disease ${ }^{10}$. In 1972 Niemeyer and Marquardt described two siblings with retinal function alteration ${ }^{11}$. They found a relative sparing in external retinal layers since the ERG was only slightly reduced, with a detectable defect in the cone than in the rod system, and elevated cone and rod thresholds on 
dark adaptation testing ${ }^{11}$. In 2005, Dedes et al. specifically noted that a patient with Wolfram syndrome had a normal ERG, "excluding tapetoretinal degeneration" as part of the differential diagnosis $^{5}$. In a large series published by Cremers and Wijdeveld et al, 88 cases of Wolfram syndrome were reviewed in the context of 3 newly diagnosed patients ${ }^{1}$. Electroretinography was performed in two of the new patients and it was described as normal in both. ${ }^{1}$ Of the 88 case reports they reviewed, only 19 underwent ERG testing, the large majority of which were normal $^{1}$. Pigmentary changes of the retina was present in $9 \%$ of the patients, but no correlation was made between ERG and fundus findings ${ }^{1}$. Barrett and Bundey analyzed 45 cases and found that none had pigmentary retinal changes ${ }^{12}$. However 6 of their patients underwent ERG testing of which $50 \%$ were normal, and the other $50 \%$ revealed a reduction in amplitude without a significant change in latency ${ }^{12}$. In a small series published from the Middle East, Al-Till et al. discussed 15 patients with Wolfram syndrome in 4 families $^{2}$. Nine of 30 eyes had retinal pigmentary changes. A normal fovea and fluorescein angiogram were documented in all patients but ERGs were not performed ${ }^{2}$. Mtanda et al. reiterated that in the first description of 11 patients with Wolfram syndrome in 1986, one had pigmentary retinal degeneration, the vast majority had some degree of cataract, and all had normal ERG and $\mathrm{EOG}^{4}$. Authors described a diminution of the dark adaptation curve characterized by a reduction in of both cone and rod adaptation ${ }^{4}$. In 2012 a report on the clinical and electrophysiological findings of 5 members of a family with WFS1 mutations showed variable degrees of changes in pattern-reversal visual evoked potentials of all patients, while dark- and light- adapted responses were of normal morphology and amplitudes on flash electroretinography ${ }^{13}$. No evidence of retinopathy was described in any of these patients ${ }^{13}$. 
Niemeyer and Marquardt assumed retrograde trans-synaptic degeneration as a possible explanation of the ERG abnormalities, causing damage to the inner nuclear layer in the central retina and subsequent reduction of the cone $\mathrm{ERG}^{11}$. Other authors considered this assumption viable to explain the abnormal ERGs findings ${ }^{3,9}$.

Given that our two patients had a normal retinal appearance in the setting of significant abnormalities in the ERG, we believe that it is possible that the retinal degeneration/dysfunction in Wolfram syndrome parallels the neurodegenerative process that underlies the disease. Although prior studies did not correlate age with onset of abnormal ERG findings, patients were examined late in their second decade or in their third decade of life, and both of our patients were in the $3^{\text {rd }}$ decade of life, so the abnormal ERGs may be a relatively late ophthalmic manifestation of Wolfram syndrome and can only be demonstrated as retinal neurons die with advancing disease. We found a normal ERG in a 9 year-old boy that we have examined at our Center. Of note, however, that Mayer et $\mathrm{al}^{3}$ presented the case of a 3.5 year-old boy diagnosed with diabetes 6 months earlier, with optic atrophy, normal fundus, and abnormal ERG, which led the authors to conclude, perhaps incorrectly, that the nature of the optic atrophy was retinal.

We would also like to posit that this retinal degeneration might be the result of mitochondrial dysfunction. Functional abnormalities in inner retinal activities examined with multifocal ERG and pattern ERG were previously described in patients with Leber's Hereditary Optic Neuropathy ${ }^{14}$. In another study, Miyata et al. described 8 patients with Autosomal Dominant Optic Atrophy caused by OPA1 mutations. They found that ERG light-adapted negative response and oscillatory potentials amplitudes were significantly reduced in the patient group $^{15}$. Although most of these indicators describe ganglion cell function, others are only partially influenced by that. In particular, the reduction in oscillatory potential amplitudes 
reflects alterations in inner plexiform layer feedback, suggesting that amacrine cells functioning could be primary or secondary related to mitochondrial dysfunction. It was also observed that oscillatory potential value decrease progressively with age ${ }^{15}$. Outer retinal abnormalities are also associated with mitochondrial diseases. Among these abnormalities, pigmentary retinopathy is the most frequent and has been associated with mtDNA mutations ${ }^{16,17}$. In 2010 Gronlund et al. studied 59 patients with a variety of mitochondrial diseases and found that $81 \%$ of cases had ophthalmologic abnormalities ${ }^{18}$. Overall, $28 \%$ of patients had abnormal retinal pigmentation. Of patients in whom ERG was performed, $37 \%$ showed retinal dystrophy with abnormalities in both a- and b-waves under dark- and light-adapted conditions ${ }^{18}$. These observations were also made in a previous paper by Zhu et al., that found pigmentary retinopathy and optic atrophy to be the most common ophthalmological manifestations in patients with confirmed mutations in well-defined primary mitochondrial diseases ${ }^{19}$.

It has been recently suggested that the mechanism leading to optic nerve atrophy in WS is similar to that in mitochondrial-associated optic neuropathies such as Leber hereditary optic neuropathy $(\mathrm{LHON})^{20}$. Although Wolframin is not a mitochondrial protein, being located in the endothelial reticulum (ER), one of its ascribed roles involves the strict ER-mitochondrial interaction in calcium exchanges. Hence Wolframin dysfunction could severely impact on calcium exchanges and mitochondrial oxidative phosphorylation leading to ER stress and apoptosis ${ }^{20}$. Because of the high reliance on mitochondrial-derived energy by neurons such as ganglion cells and photoreceptors, the correlation between mitochondrial disease and optic nerve atrophy can be explainable, and one can postulate a similar mechanism leading to photoreceptor dysfunction ${ }^{21}$. Moreover, the heteroplasmy and the threshold effect that characterize 
mitochondrial pathologies could explain why retinal dysfunction is only detected in a fraction of patients and with a delayed onset ${ }^{22}$.

In this paper, we have provided additional support to the presence of retinal dysfunction as demonstrated by abnormal electroretinography in two patients with Wolfram syndrome and postulate that these are the result of photoreceptor degeneration possibly from abnormal mitochondrial function. ERGs should be obtained in patients with Wolfram syndrome and could be repeated to document progression of the disease or its response to emerging therapies.

\section{Acknowledgements}

None

\section{Declaration of Interest}

The authors report no conflicts of interest. 


\section{$\underline{\text { References }}$}

1. Cremers CW, Wijdeveld PG, Pinckers AJ. Juvenile diabetes mellitus, optic atrophy, hearing loss, diabetes insipidus, atonia of the urinary tract and bladder, and other abnormalities (Wolfram syndrome). Acta Paediatr Scand Suppl. 1977;(264):1-16.

2. Al-Till M, Jarrah NS, Ajlouni KM. Ophthalmologic findings in fifteen patients with Wolfram syndrome. Eur J Ophthalmol. 12(2):84-8.

3. Mayer UM, Rott HD, Böhles HJ. Observation concerning the age of onset and the nature of optic atrophy in Wolfram's syndrome (DIDMOADS). Ophthalmic Paediatr Genet. 1985 Apr;5(3):155-7.

4. Mtanda AT, Cruysberg JR, Pinckers AJ. Optic atrophy in Wolfram syndrome. Ophthalmic Paediatr Genet. 1986 Dec;7(3):159-65.

5. Dedes W, Wildberger H, Landau K. Optic atrophy as a sign of wolfram syndrome. Klin Monbl Augenheilkd. 2005 Mar;222(3):248-51.

6. Barrett TG, Bundey SE, Macleod AF. Neurodegeneration and diabetes: UK nationwide study of Wolfram (DIDMOAD) syndrome. Lancet (London, England). 1995 Dec 2;346(8988):1458-63.

7. Barrett TG. Differential diagnosis of type 1 diabetes: which genetic syndromes need to be considered? Pediatr Diabetes. 2007 Oct;8 Suppl 6(s6):15-23.

8. Cryns K, Sivakumaran TA, Van den Ouweland JMW, Pennings RJE, Cremers CWRJ, Flothmann K, et al. Mutational spectrum of the WFS1 gene in Wolfram syndrome, nonsyndromic hearing impairment, diabetes mellitus, and psychiatric disease. Hum Mutat. 2003 Oct;22(4):275-87.

9. Gunn T, Bortolussi R, Little JM, Andermann F, Fraser FC, Belmonte MM. Juvenile 
diabetes mellitus, optic atrophy, sensory nerve deafness, and diabetes insipidus--a syndrome. J Pediatr. 1976 Oct;89(4):565-70.

10. Rose FC, Fraser GR, Friedmann AI, Kohner EM. The association of juvenile diabetes mellitus and optic atrophy: clinical and genetical aspects. Q J Med. 1966 Jul;35(139):385405 .

11. Niemeyer G, Marquardt JL. Retinal Function in an Unique Syndrome of Optic Atrophy, Juvenile Diabetes Mellitus, Diabetes Insipidus, Neurosensory Hearing Loss, Autonomic Dysfunction, and Hyperalanineuria. Invest Ophthalmol Vis Sci. 1977 Jul 1;11(7):617-24.

12. Barrett TG, Bundey SE, Fielder AR, Good PA. Optic atrophy in Wolfram (DIDMOAD) syndrome. Eye (Lond). 1997 Nov 1;11 ( Pt 6)(6):882-8.

13. Langwińska-Wośko E, Broniek-Kowalik K, Szulborski K. A clinical case study of a Wolfram syndrome-affected family: pattern-reversal visual evoked potentials and electroretinography analysis. Doc Ophthalmol. 2012 Apr 7;124(2):133-41.

14. Kurtenbach A, Leo-Kottler B, Zrenner E. Inner retinal contributions to the multifocal electroretinogram: patients with Leber's hereditary optic neuropathy (LHON). Multifocal ERG in patients with LHON. Doc Ophthalmol. 2004 May;108(3):231-40.

15. Miyata K, Nakamura M, Kondo M, Lin J, Ueno S, Miyake Y, et al. Reduction of Oscillatory Potentials and Photopic Negative Response in Patients with Autosomal Dominant Optic Atrophy with OPA1 Mutations. Investig Opthalmology Vis Sci. 2007 Feb $1 ; 48(2): 820$.

16. Mullie MA, Harding AE, Petty RKH, Ikeda H, Morgan-Hughes JA, Sanders MD. The Retinal Manifestations of Mitochondrial Myopathy. Arch Ophthalmol. 1985 Dec 1;103(12):1825. 
17. Al-Enezi M, Al-Saleh H, Nasser M, Al-Saleh H, MD, FRCS, et al. Mitochondrial disorders with significant ophthalmic manifestations. Middle East Afr J Ophthalmol. 2008 Apr;15(2):81-6.

18. Gronlund MA, Honarvar AKS, Andersson S, Moslemi AR, Oldfors A, Holme E, et al. Ophthalmological findings in children and young adults with genetically verified mitochondrial disease. Br J Ophthalmol. 2010 Jan 1;94(1):121-7.

19. Zhu C-C, Traboulsi EI, Parikh S. Ophthalmological findings in 74 patients with mitochondrial disease. Ophthalmic Genet. 2017 Jan 2;38(1):67-9.

20. Ross-Cisneros FN, Pan XP, Silva RA, Miller NR et al., Optic nerve histopathology in a case of Wolfram Syndrome: A mitochondrial pattern of axonal loss, Mitochondrion (2013)

21. Linsenmeier RA. Effects of light and darkness on oxygen distribution and consumption in the cat retina. J Gen Physiol. 1986 Oct;88(4):521-42.

22. Moggio M, Colombo I, Peverelli L, Villa L, Xhani R, Testolin S, et al. Mitochondrial disease heterogeneity: a prognostic challenge. Acta Myol myopathies cardiomyopathies Off J Mediterr Soc Myol. 2014 Oct;33(2):86-93.

23. McCulloch DL, Marmor MF, Brigell MG, Hamilton R, Holder GE, Tzekov R, et al. ISCEV Standard for full-field clinical electroretinography (2015 update). Doc Ophthalmol. 2015 Feb $14 ; 130(1): 1-12$. 
Table 1. Literature about ERG abnormalities findings in patients with Wolfram Syndrome

\begin{tabular}{|c|c|c|c|}
\hline \multirow[t]{2}{*}{ Reference } & \multicolumn{3}{|r|}{ Patient } \\
\hline & Age and Sex & Macula & ERG Characteristics \\
\hline \multirow[t]{3}{*}{ Gunn et al. ${ }^{9} 1976$} & $17 \mathrm{~F}$ & Normal & $\begin{array}{l}\text { Dark-adapted elevated threshold and diminished amplitude } \\
\text { Light-adapted normal response } \\
\text { Dark- and light-adapted diminished oscillatory potential } \\
\text { Abnormal dark adaptation }\end{array}$ \\
\hline & $17 \mathrm{~F}$ & Normal & $\begin{array}{l}\text { Dark-adapted elevated threshold and diminished amplitude } \\
\text { Light-adapted elevated threshold and diminished amplitude } \\
\text { Dark- and light-adapted diminished oscillatory potential } \\
\text { Abnormal dark adaptation }\end{array}$ \\
\hline & $17 \mathrm{M}$ & Normal & Light-adapted elevated threshold and diminished amplitude \\
\hline \multirow[t]{2}{*}{ Niemeyer at al. ${ }^{11} 1977$} & $19 \mathrm{~F}$ & Normal & $\begin{array}{l}\text { Dark- and light-adapted very slightly reduced amplitudes } \\
\text { No significant change in latency or implicit time } \\
\text { Dark- and light-adapted diminished oscillatory potential } \\
\text { Dark- and light-adapted elevated threshold at dark adaptation }\end{array}$ \\
\hline & $16 \mathrm{M}$ & Normal & $\begin{array}{l}\text { Dark- and light-adapted very slightly reduced amplitudes } \\
\text { No significant change in latency or implicit time } \\
\text { Dark- and light-adapted diminished oscillatory potential } \\
\text { Dark- and light-adapted elevated threshold at dark adaptation }\end{array}$ \\
\hline Barrett et al. $^{12} 1997$ & $\begin{array}{l}\text { Not known } \\
\text { (3 patients) }\end{array}$ & Normal & $\begin{array}{l}\text { Dark- and light-adapted reduction in amplitude of response } \\
\text { without significant change in latency }\end{array}$ \\
\hline \multirow[t]{2}{*}{ Our study } & $22 \mathrm{~F}$ & Normal & Dark- and light-adapted reduction in amplitude of response \\
\hline & $26 \mathrm{M}$ & Normal & Dark- and light-adapted reduction in amplitude of response \\
\hline
\end{tabular}




\section{Caption}

Figure 1. Combined images of Case 1. Right eye (A-B) and left eye (C-D) fundus photographs and fundus autofluorescence of a 22 years old woman show mild disc pallor in both eyes, but normal fundus appearance.

Figure 2. Full-field ERGs for Case I and Case II. Nomenclature follows the terminology proposed by latest ISCEV update on full-field electroretinography ${ }^{23}$. Dark-adapted -24 ERG (DA -24), Dark-adapted 0 ERG (DA 0), Light-adapted 0 ERG (LA 0) and Lightadapted $30 \mathrm{~Hz}$ Flicker (LA $30 \mathrm{~Hz}$ ) Recorded Using DTL Electrodes in a Control Case, in Case 1 (S1) and in Case 2 (S2). Low luminance stimulus evoked a response with reduced amplitude in b-wave in both patients under dark-adapted conditions (top row). In response to a high luminance stimulus (row 2), the a-wave amplitude was also reduced. Under light-adapted conditions that isolate cone responses, ERG amplitudes were reduced for both single flash and flicker stimuli (Rows 3, 4).

Figure 3. Right and left (A-B) eye fundus photographs of a 26 years old man show marked disc pallor, without fundus abnormalities 